cedure becomes established children who are found at the routine school eye test to have defects of vision will already have been identified and be under treatment, except, of course, for those missed in the initial screening. But they will be no worse off than they are at the moment and it is hoped that a considerable number of children who are being overlooked at present will benefit from the early treatment of their defects. It seems much more profitable to devote resources to a screening procedure of this sort than to the somewhat outdated task of purveying spectacle prescriptions at traditional school eye clinics.

H CAMERoN

Ophthalmic Unit,

Ayr

\section{Evolution of poliovirus since introduction of attenuated vaccine}

SIR,-Dr H V Wyatt (20 August, p 518) seeks the elusive paralytic rate of poliomyelitis with neurological manifestations in the period before the era of oral vaccination. Figures from the collaborative survey designed to study all cases of typical and suspected poliomyelitis, including non-paralytic "aseptic meningitis," in the Western and Eastern Hospital Board areas of Scotland during the first two years of introduction of inactivated vaccine may be helpful. ${ }^{1}$

Polioviruses were isolated from $79(71 \%)$ of 112 patients classified clinically as paralytic poliomyelitis and from $26(11 \%)$ of 239 with non-paralytic meningitis. Thus 79 of 105 poliovirus infections with neurological disease were paralytic-a paralysis rate of $75 \%$ Imperfections of the virus isolation procedure mean that the true rate may deviate from this estimate, but probably not by much: serological tests in parallel revealed only 16 additional poliovirus infections." By similar calculations the paralytic rates were over $60 \%$ in $1958-9,{ }^{3}$ the last major epidemic in this area, and $74 \%$ in $1962,{ }^{4}$ the last outbreak before control was achieved by the general use of oral vaccine.

University Department of

Infectious Diseases,
Ruchill Hospital,

Glasgow

1 Grist, N R, et al, Health Bulletin, 1958, 16, 27

Grist, N R, et al, Scottish Medical fournal, 1960, 5,

Sommerville, R G, MD thesis, University of Glasgow, 1959.

- Green, D M, and Bell, E J, Ninth Symposium of European Association against Poliomyelitis and Allied Diseases,
Masson, 1964.

\section{Atherosclerosis, Down's syndrome, and} Alzheimer's disease

SIR,-In their very interesting paper Dr J C Murdoch and others (23 July, p 226) report a complete absence of atherosclerosis in all of five patients with Down's syndrome who went to necropsy. At the same time they report that the cause of death in 11 of 21 subjects not necropsied had been reported as cardiovascular in origin.

Some years ago I came across a similar situation in regard to Alzheimer's disease (senile psychosis, cerebral amyloidosis). Here too most of these cases are attributed to "cerebral arteriosclerosis" during life. Post- mortem examination, however, invariably showed minimal atherosclerosis and occasionally complete absence of this disease. I was often astonished to note "lily-white" arteries in subjects over 80 years of age who had suffered from Alzheimer's disease during life.

Cerebral amyloidosis has been reported as a very common finding in elderly-aged 35 years and over-mongols. It would be very interesting to know if in fact Dr Murdoch and his colleagues found many plaques or tangles in their cases. Such a finding would be of great interest to those trying to probe the causation and mechanism of Alzheimer's disease.

Bristol

William Hughes

${ }^{*}{ }_{*}^{*}$ We sent a copy of this letter to Dr Murdoch, who consulted Professor Hume Adams, who carried out the neuropathological studies in the cases referred to. Their joint reply is printed below.-ED, $B M \mathcal{~ F}$.

SIR,-Four of the five subjects who went to necropsy had the brain submitted for neuropathological examination to the Institute of Neurological Sciences, Glasgow. In three (men aged 40 and 60 and a woman aged 66) there were large numbers of plaques in the representative areas of cortex examined, but in only two of these were there numerous examples of neurofibrillary change. In the fourth case (man aged 56) there were no significant plaques or examples of neurofibrillary change. These findings would accord with the previously reported high incidence of Alzheimer's disease in elderly subjects with Down's syndrome.

Department of General Practice,

J C MuRdoch University of Dundee

\section{University Department of}

Hume ADAMS

\section{Neuropathology}

Institute of Neurological Sciences,

Southern General Hospital,

Glasgow

\section{Medical genetics}

SIR,-Your readers may wish to know that copies of the memorandum on the training of medical geneticists (leading article, 30 July, p 279) prepared by the working party of the Clinical Genetics Society may be obtained from the undersigned.

A W Johnston

Woodend General Hospital, Chairma

Aberdeen

\section{Immersion and drowning in children}

SIR,-Since the subject of airway warming in the treatment of accidental hypothermia has been raised in your leading article (16 July, $p$ 146) and the subsequent letter from Dr C D Auld and Professor J N Norman (20 August, p 519) I feel that it is time to get the facts in perspective.

In my original article ${ }^{1}$ it was suggested that if a non-shivering hypothermic person breathed an atmosphere which is warm and humid it might be possible to conserve $30 \%$ of the metabolic heat produced. On theoretical ${ }^{12}$ and experimental grounds ${ }^{3}$ airway warming is only a supplement to insulation of the body. Nevertheless, in animal experiments ${ }^{3}$ and clinical cases ${ }^{2}$ airway warming has been shown to accelerate the rate of rewarming as compared with surface insulation alone. (This fact makes it potentially dangerous for continuous use in chronic hypothermia, ${ }^{4}$ for example, in the elderly if the patients are allowed to breathe spontaneously.) Though the rate of rewarming is of interest there are other factors important in the treatment of hypothermia. Airway warming appears to have a beneficial effect on cardiovascular status ${ }^{25}$ and cardiac rhythm ${ }^{26}$ out of proportion to the actual rise in temperature, and clinical observations suggest a similar improvement in cerebral function. ${ }^{2}$ In spite of these possible benefits a lot of research is still required to establish the clinical value of airway warming and to allow its use in the treatment of accidental hypothermia to be accepted or rejected.

Since airway warming can act only as a supplement to surface insulation any equipment should be fail-safe, and this means, in addition to other factors, that the initial heat/ moisture surge of the $\mathrm{CO}_{2} /$ soda lime reaction ${ }^{7}$ must be controlled. This is especially important since the equipment will be used infrequently by the non-medical members of the rescue services, usually under adverse conditions. I can therefore only repeat ${ }^{8}$ that, to my knowledge, though a number of designs have been described (some commercially available) and while these may be effective in the hands of their originators, there is still insufficient information available to allow any unit to be recommended for general use.

The equipment initially described ${ }^{1}$ is not the Reviva despite the reference in the letter from Dr Auld and Professor Norman, and in fact the Reviva is a totally different design.

Department of Anaesthetics,

E LL LLOYD Royal Infirmary,

${ }^{1}$ Lloyd, E L, et al, Scottish Medical Fournal, 1972, 17, 83.
4loyd, E L, British fournal of Anaesthetics, 1973, 45,

Lloyd, E L, Mitchell, B, and Williams, J T, Resuscitation, 1976, 5, 5 .

5 Lloyd, E L, Mitchell, B, and Williams, J T, Resuscita-

tion. In press.
6 Lloyd, E L, and Mitchell, B, Lancet, 1974, 2, 1294. Guild, W J, fournal of the Royal Naval Medical Service, 1976, 62, 173.
Lloyd, E L, Outdoors, 1977, 8, 9.

\section{Doctors' errors}

SIR,-In a recent leading article ( 7 May, $\mathrm{p}$ 1178) on reducing doctors' errors it was suggested that medicine of the future might include a computer-aided system sitting on the physician's shoulder. In the present state of the economy this would seem to be a pipe-dream and it is often rewarding to look at simple, cheaper alternatives which might go some way to reduce errors.

The device described here was developed for use in general practice, but that is not to say that its use is limited to that setting. The idea of the device was occasioned by my dislike of a too rigid concept of the decisiontree approach to diagnosis and treatment while being only too painfully aware that the amount of information available to bear on a given case was often so great that it might not be mobilised every time it was needed and that the growing complexity of interactions between different factors has become difficult to bear constantly in mind.

As there are dual pathways of diagnosis and 
treatment to monitor, as well as the consideration of keeping the whole device small and easily assimilable, the idea of a clock-face layout surrounding the current medical record card seemed the most economical (see figure).

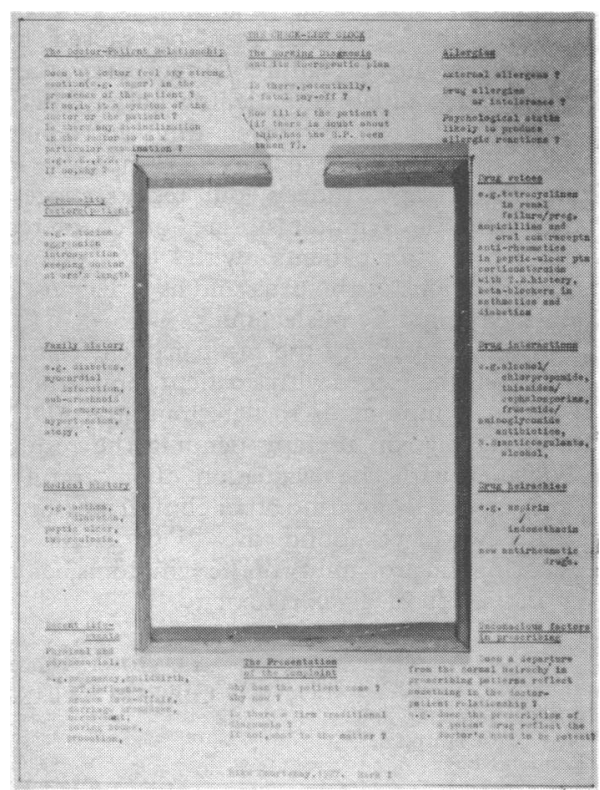

The check-list clock.

There are twelve headings, six to do with the diagnostic process, four to do with the diagnostic process, and two involving both. They are as follows.

Presentation of the complaint. (Why has the patient come? Why now ? Is there a firm traditional diagnosis? If not, what is the matter?)

Medical history.

Family history.

Recent life events. (For example, childbirth, marriage breakdown, bereavement, etc.)

Personality factors. (For example, stoicism, introspection, aggression, keeping doctor at arm's length.)

Doctor-patient relationship. (Does the doctor feel any strong emotion, such as anger, in the presence of the patient ? If so, is it a symptom of the doctor or the patient? Is there any disinclination of the doctor to do a particular examination (for example, vaginal, rectal) ? If so, why ?)

Allergies. (Any external allergies, drug allergies or intolerances, psychological state likely to produce reactions to treatment ?)

Drug vetoes. (Common examples listed.)

Drug interactions. (Common examples listed.)

Drug hierarchies. (For example, aspirin-indomethacin-newer antirheumatic drugs.)

Unconscious factors in prescribing. (Does a departure from the normal (strength) hierarchy in prescribing patterns reflect something in the doctor-patient relationship ? For example, does the prescription of a potent drug reflect the doctor's need to be potent with that particular patient or does the prescription of a cheap preparation reflect the doctor's contempt?)

Working diagnosis and its therapeutic plan. (Is there, potentially, a fatal pay-off? How ill is the patient? (If there is doubt, has the blood pressure been taken ?))

Initial experience with the check-list clock, placing the current medical record continuation card in the central part of the frame, has shown that this kind of review of the consultation conducted initially in an unstructured way has led to important changes in both diagnosis and treatment through the stimulation of further recall and understanding of important information and interaction which had previously been missed.

\section{When is significant not significant?}

SIR,-The work of Sir Richard Doll and $\mathrm{Mr}$ Richard Peto (4 June, p 1433) raises several points of interest.

With figs 1 and 2 the reader is asked to bias his judgment in favour of a relation which the authors appear intent on proving; the broken lines used for five confidence intervals enforce this bias. However, the basis for this choice of "reliable" and "unreliable" SMRs is not indicated. Definition of statistical methods being poor, the authors would appear to have calculated regression lines using 11 pairs of variables. However, these calculations (using, presumably, the mean values for each specialty) fail to take into account the often large scatter of data about each group mean, this influencing in turn the error associated with the estimation of regression line gradients. Thus in using apparently grouped data for their calculations the authors would seem to be biasing the analysis, again in favour of demonstrating a significant relation between variables.

In their subsequent letter $\mathrm{Mr}$ Peto and Professor Doll (23 July, p 259) state that "moderate statistical significance (such as $\mathbf{P}<0.05$ ) [in association] often does arise as an artefact of chance." This statement is worthless. From the statistical estimation one is able to suggest that the observed data would have occurred by chance in less than one case in 20 if one is willing to accept the predefined risk of erroneous conclusion, then one can but conclude that certain relations have, or have not, been demonstrated in the sample group. It is irrelevant whether a causal relation can be established. The authors fail to define their level of significance before the analysis of their observations; as a consequence of this omission Professor Doll and Mr Peto in their paper appear to have succumbed to the serious error of choosing only those statistical estimations which suit their purpose, as suggested by Professor Hugh Dudley (2 July, p 47). Moreover, they conclude that "the excess of ischaemic heart disease and chronic bronchitis in single-handed general practitioners and the deficiency of ischaemic heart disease in hospital physicians and surgeons, and of chronic bronchitis in hospital physicians, may be attributed respectively to their above and below average consumption of cigarettes" (my italics); herein lies the common error of implicating causal relations between variables linked only by statistical estimation.

The example of throwing paired dice, as given by Mr Peto and Professor Doll in their letter, although correct probability theory, is an example of poor logic and misuse of statistical estimation. Of course the probability of throwing a double-six is about 0.03 ; however, this does not even suggest that the dice are biased because the probability of throwing any pair of numbers (for example, 4 on dice $A$ and 1 on dice $B$ ) is about 0.03 . The very suggestion that the judgment should be made on the basis of a single throw implies a failure to grasp an elementary principle of statisticsnamely, that statistical estimations can be made only on the basis of populations of figures. The words of a great statistician, the late Sir Ronald Fisher, would be of undoubted value here: ". . . Nevertheless, in a real sense statistics is the study of populations, or aggregates of individuals, rather than of individuals. Scientific theories which involve the properties of large aggregates of individuals, and not necessarily the properties of the individuals themselves ... . are essentially statistical argu- ments, and are liable to misinterpretation as soon as the statistical nature of the argument is lost sight of."

King's College Hospital,

Geoffrey E Rose

King's College
London SE5

Hallucinations in polymyalgia arteritica

SIR,-Polymyalgia arteritica has been commendably treated in some of your recent issues. One feature I did not observe: the occurrence of hallucinations in connection with this disease. During the last few years that I was head of the Medical University Clinic in Uppsala, we saw some 50 cases of polymyalgia arteritica. Visual hallucinations were not common but did occur: an old lady came into the clinic escorted by a St Bernard dog which was entirely fictitious. She wanted the dog placed in the bed next to her and this permission was of course granted. The following morning, after she had received, the previous day, a rather massive dose of cortisone, the the dog had disappeared, much to her embarrassment. The subsequent treatment kept the dog away from her.

ERIK ASK-UPMARK

Uppsala, Sweden

\section{Congenital dislocation of the hip}

SIR,-With reference to $\mathrm{Mr}$ A J Harrold's excellent review of the problems in congenital dislocation of the hip (CDH) (23 April, p 1071) I would like to present a deviating opinion on a few points.

It is true that the incidence of $\mathrm{CDH}$ has increased in those clinics which have introduced the technique of Ortolani and Barlow in examining all newborn infants. It is not true, however, that this increase of incidence is due to "overdiagnosis," including completely healthy infants, as it has been demonstrated ${ }^{1}$ that the condition in these children is closely related to $\mathrm{CDH}$. All these children have unstable hips, some of which are dislocated or dislocatable at the moment of examination. There are no means, however, of distinguishing between those hips which will recover spontaneously and those in which complete dislocation will develop later on.

Should all these children be treated and is treatment harmless? Not only failures but also a dangerously high complication rate in the treatment of $\mathrm{CDH}$ have been reported. ${ }^{2-4}$ All these misfortunes, however, have occurred in children who have either been treated late or with rigid fixation. In a long-term followup $^{5}$ 8-16 years after treatment it has been demonstrated that proper treatment is completely harmless.

Acetabular dysplasia is a common finding among the parents of children with $\mathrm{CDH} .^{6}$ A child with a minor degree of hip instability that will allow only partial dislocation (subluxation) can remain symptom-free throughout childhood. The resulting incongruence will lead to underdevelopment of the acetabular roof (dysplasia) ${ }^{7}$ and perhaps to early arthritis. ${ }^{8-11}$ Treatment in a harmless splint for a few months will probably prevent this course.

The literature on the difficulties of early diagnosis and treatment is abundant. During the past few years, however, it has become evident that the difficulties can be overcome and by 